MATHEMATICS OF COMPUTATION

Volume 79, Number 270, April 2010, Pages 829-844

S 0025-5718(09)02279-0

Article electronically published on August 26, 2009

\title{
FAST INTEGRATION OF HIGHLY OSCILLATORY INTEGRALS WITH EXOTIC OSCILLATORS
}

\author{
SHUHUANG XIANG AND HAIYONG WANG
}

\begin{abstract}
In this paper, we present an efficient Filon-type method for the integration of systems containing Bessel functions with exotic oscillators based on a diffeomorphism transformation and give applications to Airy transforms. Preliminary numerical results show the effectiveness and accuracy of the quadrature for large arguments of integral systems.
\end{abstract}

\section{INTRODUCTION}

In many areas of applied mathematics one encounters the problem of computing rapidly oscillatory integrals of the type

$$
I[f]=\int_{a}^{b} f(x) S(\omega g(x)) d x
$$

where $S$ is an oscillatory function, $f(x)$ and $g(x)$ are sufficiently smooth functions, $\omega$ is a large parameter and $a$ and $b$ are real and finite. In most of the cases, such transforms cannot be calculated analytically and one has to resort to numerical methods. When the integrand becomes highly oscillatory, it presents serious difficulties in obtaining numerical convergence of the integration.

Many efficient methods for computing (1.1) when $S(\omega g(x))=e^{i \omega g(x)}$, where $g^{\prime}(x) \neq 0$ for all $x \in[a, b]$, have been devised. See, for example, the Filon method [2, [5, 6, 16, the Levin method [14, generalized quadrature rules [3, 4, the asymptotic method [11, the Filon-type method [10, 11, 25, the Levin-type method [17] and the steepest descent method [8]. When $S$ is a Bessel function of the first kind $J_{v}(\omega x)$, there are also a few methods available. For example, the modified Clenshaw-Curtis method [20, 21] is efficient for computing $\int_{0}^{1} f(x) J_{\nu}(\omega x) d x$ for nonnegative orders $\nu$; the Levin-type method [15, 18, 26, 27] and generalized quadrature rules [3, 4, 28] are efficient for approximating $\int_{a}^{b} f(x) J_{\nu}(\omega x) d x$ for $\operatorname{Re}(\nu)>-1$ and $0 \notin[a, b]$. Moreover, the Levin-type method and generalized quadrature rules can be extended to approximate the integral $\int_{a}^{b} f(x) J_{v}(\omega g(x)) d x$ if $g(x) \neq 0$ and $g^{\prime}(x) \neq 0$ for all $x \in[a, b]$.

Received by the editor December 28, 2007 and, in revised form, October 25, 2008 and March $18,2009$.

2000 Mathematics Subject Classification. Primary 65D32, 65D30.

Key words and phrases. Oscillatory integrals, diffeomorphism transformation, Filon-type method.

This work is supported by NSF of China (No.10771218) and the Program for New Century Excellent Talents in University, State Education Ministry, China.

(C)2009 American Mathematical Society Reverts to public domain 28 years from publication 
Once $g^{\prime}$ vanishes at one or more points in $[a, b]$, which are known as critical points, the above numerical evaluation may be difficult. For treatment of critical points for $\int_{a}^{b} f(x) e^{i \omega g(x)} d x$, without loss of generality, assume $\xi_{0} \in[a, b]$ is the unique turning point of $g$ and for an integer $r \geq 1$,

$$
\begin{aligned}
g^{\prime}\left(\xi_{0}\right)=g^{\prime \prime}\left(\xi_{0}\right)=\cdots= & g^{(r)}\left(\xi_{0}\right)=0, g^{(r+1)}\left(\xi_{0}\right) \neq 0 \\
& \text { and } g^{\prime}(x) \neq 0 \text { for } x \neq \xi_{0}, x \in[a, b] .
\end{aligned}
$$

From Stein ([22, p. 334), it is known from the classical method of the stationary phase that for every smooth function $f$ with compact support near $\xi_{0}$, there exist linear differential functionals $A_{k}, k=0,1, \ldots$, such that

$$
I[f] \sim \omega^{-1 /(r+1)} \sum_{k=0}^{\infty} A_{k}[f] \omega^{-k /(r+1)}, \quad \omega \gg 1 .
$$

The following asymptotic expansion developed by Iserles and Nørsett [1] provides an invaluable tool for computing highly oscillatory integrals:

$$
\begin{aligned}
I[f] \sim & \sum_{j=0}^{r-1} \frac{1}{j !} \mu_{j}\left(\omega, \xi_{0}\right) \sum_{k=0}^{\infty} \frac{1}{(-i \omega)^{k}} \rho_{k}^{(j)}[f]\left(\xi_{0}\right) \\
& -\sum_{k=1}^{\infty} \frac{1}{(-i \omega)^{k}}\left(\frac{e^{i \omega g(b)}}{g^{\prime}(b)}\left\{\rho_{k-1}[f](b)-\sum_{j=0}^{r-1} \frac{\rho_{k-1}[f]^{(j)}\left(\xi_{0}\right)(b-\xi)^{j}}{j !}\right\}\right. \\
& \left.-\frac{e^{i \omega g(a)}}{g^{\prime}(a)}\left\{\rho_{k-1}[f](a)-\sum_{j=0}^{r-1} \frac{\rho_{k-1}[f]^{(j)}\left(\xi_{0}\right)(a-\xi)^{j}}{j !}\right\}\right), \quad \omega \gg 1,
\end{aligned}
$$

where $\mu_{j}\left(\omega, \xi_{0}\right)=\int_{a}^{b}\left(x-\xi_{0}\right)^{j} e^{i \omega g(x)} d x, j=0,1, \ldots, r-1$, and

$$
\rho_{0}[f](x)=f(x), \quad \rho_{k+1}[f](x)=\frac{d}{d x} \frac{\rho_{k}[f](x)-\sum_{j=0}^{r-1} \frac{1}{j !} \rho_{k}[f]^{(j)}\left(\xi_{0}\right)\left(x-\xi_{0}\right)^{j}}{g^{\prime}(x)}, k \geq 0 .
$$

The generalized asymptotic method (the finite sums of the infinite series (1.3)) and the generalized Filon-type method presented in [11] are efficient in dealing with highly oscillatory integrals involving critical points under the condition that the first few moments

$$
\mu_{k}\left(\omega, \xi_{0}\right)=\int_{a}^{b}\left(x-\xi_{0}\right)^{k} e^{i \omega g(x)} d x, \quad k=0,1, \ldots, r-1,
$$

are in explicit forms. Unfortunately, the moments are often unknown. This situation is readily remedied by a Filon-type method recently developed by Olver [19, where the generalized moments for the basis functions can be computed explicitly by the solutions of the collocation differential equations

$$
u^{\prime}+i \omega g^{\prime}(x) u=x^{k}, \quad k=0,1, \ldots
$$

However, it is difficult to extend this method to approximate Bessel or Airy transforms since in these cases the corresponding collocation differential equations cannot be solved exactly.

The critical case for $S(\omega g(x))=e^{i \omega g(x)}$ was also handled in Huybrechs and Vandewalle [8] by going to the complex plane, and integrating along a path that 
approximated the path of steepest descent. Numerical steepest descent methods for evaluating the integrals involving Hankel functions of the form

$$
\int_{a}^{b} f(x) H_{\nu}^{(1)}\left(\omega g_{1}(x)\right) e^{i \omega g_{2}(x)} d x
$$

were proposed in [9]. Since the Bessel function is the real part of the Hankel function and decays exponentially in the complex plane, then numerical steepest descent methods can also be used to evaluate the integrals involving the Bessel function. However, these methods require the functions $f, g_{1}$ and $g_{2}$ should be analytic in the complex plane containing $[a, b]$.

The purpose of this paper is to solve the open problems discussed in Iserles et al. [12, 13] and Olver [18, regarding the efficient computation of the integrals

$$
\int_{0}^{1} f(x) J_{m}(\omega g(x)) d x, \quad \int_{0}^{1} f(x) A i(-\omega g(x)) d x
$$

with the exotic oscillator $g(x)$ that satisfies for $r \geq 0$,

$$
g(0)=g^{\prime}(0)=\cdots=g^{(r)}(0)=0, \quad g^{(r+1)}(0) \neq 0, \quad g^{\prime}(x) \neq 0 \text { for } x \in(0,1],
$$

where $\operatorname{Re}(m)>-\frac{1}{r+1}$. (For $\operatorname{Re}(m) \leq-\frac{1}{r+1}$, in general, the integral (1.7) is not defined. Consider $\int_{0}^{1} \sin (x) J_{-0.5}\left(r x^{2}\right) d x$ as an example.) Without loss of generality, we assume $\omega>0$ and $g^{(r+1)}(0)>0$ (replacing $g$ by $-g$ if necessary). From (1.7) we see that $g(x)$ is strictly monotonic on $[0,1]$ and $g(x)>0$ for $x \in(0,1]$. Following [25], we apply a diffeomorphism transformation $t^{r+1}=g(x)$ for the exotic oscillator of the integrand $\int_{0}^{1} f(x) S(\omega g(x)) d x$ such that the moments $I\left[t^{k}\right]=\int_{0}^{r+1} \sqrt{g(1)} t^{k} S\left(\omega t^{r+1}\right) d t$ can be calculated explicitly. Then we can compute the resulting integrals efficiently.

The rest of the paper is organized as follows. In Section 2 we introduce a Filontype method for (1.6) and present explicit expressions for the moments of the Bessel and Airy transforms. The details of the computation of Lommel functions are also discussed there. In Section 3 we first present the asymptotic expansion for the Bessel transform $\int_{0}^{1} f(x) J_{m}(\omega x) d x$, and then we give the error analysis of the Filon-type method for $\int_{a}^{b} f(x) J_{m}(\omega g(x)) d x$. Preliminary numerical examples show that the Filon-type method is efficient and accurate for approximating the integral considered. Finally, we consider applications to Airy transforms in Section 4.

A word about our notation. (i) If $f(x) / \phi(x)$ tends to unity as $x \rightarrow x_{0}$, we write

$$
f(x) \sim \phi(x) \quad\left(x \rightarrow x_{0}\right) .
$$

In words, $f$ is asymptotic to $\phi$.

(ii) If $f(x) / \phi(x) \rightarrow 0$ as $x \rightarrow x_{0}$, we write

$$
f(x)=o(\phi(x)) \quad\left(x \rightarrow x_{0}\right) .
$$

(iii) If $f(x) / \phi(x)$ is bounded as $x \rightarrow x_{0}$, we write

$$
f(x)=O(\phi(x)) \quad\left(x \rightarrow x_{0}\right) .
$$




\section{Filon-type method And EFFiCiEnt EVAluation of the MOMENTS}

We investigate the following highly oscillatory integral:

$$
I[f]=\int_{0}^{1} f(x) S(\omega g(x)) d x
$$

where $f(x)$ and $g(x)$ are smooth functions and $g(x)$ satisfies $(1.7)$ with $g^{(r+1)}(0)>0$.

Let $t^{r+1}=g(x)$. Then from Stein $([22$, pp. 336-337), $t$ is well defined in $[0,1]$ and is a diffeomorphism in a small neighborhood of 0 . Thus $t$ is a diffeomorphism in $[0,1]$ since $g^{\prime}(x) \neq 0$ for $x \in(0,1]$. Therefore, $x(t)$ is well defined and $I[f]$ can be rewritten as

$$
\begin{aligned}
I[f]=\int_{0}^{1} f(x) S(\omega g(x)) d x & =(r+1) \int_{0}^{y_{0}} \frac{f(x) g(x)^{r /(r+1)}}{g^{\prime}(x)} S\left(\omega t^{r+1}\right) d t \\
& =(r+1) \int_{0}^{y_{0}} \tilde{f}(t) S\left(\omega t^{r+1}\right) d t
\end{aligned}
$$

where $\tilde{f}(t)=\frac{f(x) g(x)^{r /(r+1)}}{g^{\prime}(x)}$ and $y_{0}=\sqrt[r+1]{g(1)}$.

Filon-type method. Let $\left\{c_{j}\right\}_{j=1}^{v}$ be a set of node points such that $0=c_{1}<c_{2}<$ $\cdots<c_{v}=1$ and denote

$$
d_{j}=\sqrt[r+1]{g\left(c_{j}\right)}, \quad j=1,2, \ldots, v
$$

Assume that $s$ is a nonnegative integer and $\left\{m_{k}\right\}_{1}^{v}$ is a set of multiplicities associated with the node points $0=d_{1}<d_{2}<\cdots<d_{v}=y_{0}$ such that $m_{1}=s(r+1)+k_{0}$ $\left(0 \leq k_{0} \leq r, m_{1} \geq 1\right)$ and $m_{v} \geq s$. Suppose that $p(t)=\sum_{k=0}^{n} a_{k} t^{k}$, where $n=\sum_{k=1}^{v} m_{k}-1$ is the solution of the system of equations

$$
p\left(d_{k}\right)=\widetilde{f}\left(d_{k}\right), p^{\prime}\left(d_{k}\right)=\widetilde{f}^{\prime}\left(d_{k}\right), \ldots, p^{\left(m_{k}-1\right)}\left(d_{k}\right)=\widetilde{f}^{\left(m_{k}-1\right)}\left(d_{k}\right),
$$

for every integer $1 \leq k \leq v$. Then the Filon-type method is defined as follows:

$$
Q_{s}^{F}[f]=(r+1) \int_{0}^{y_{0}} p(t) S\left(\omega t^{r+1}\right) d t=(r+1) \sum_{k=0}^{n} a_{k} I\left[t^{k}\right],
$$

where $I\left[t^{k}\right]=\int_{0}^{y_{0}} t^{k} S\left(\omega t^{r+1}\right) d t$. For $d_{k} \in\left[0, y_{0}\right], k=1,2, \ldots, v$, we obtain $\widetilde{f}\left(d_{k}\right)=$ $\frac{f\left(c_{k}\right) g\left(c_{k}\right)^{r /(r+1)}}{g^{\prime}\left(c_{k}\right)}$. The derivatives of $\widetilde{f}^{(\ell)}(t)$ can be computed by

$$
\frac{d^{\ell}\left[\frac{f(x) t^{r}}{g^{\prime}(x)}\right]}{d t^{\ell}}
$$

where $x$ is considered as a function of $t$ and $x^{\prime}(t)=\frac{(r+1) t^{r}}{g^{\prime}(x)}$. In particular, for $d_{0}=0, \tilde{f}(0)$ and $\tilde{f}^{(k)}(0)$ are computed by their limits using the Taylor expansion of $g(x)$. In this paper we choose the quadrature points as the shifted Chebyshev points $c_{k}=\frac{1+\cos \left(\frac{(v-k) \pi}{v-1}\right)}{2}, k=1, \ldots, v$. 
- For $S\left(\omega t^{r+1}\right)=J_{m}\left(\omega t^{r+1}\right)$ : If $\omega>0$ and $\operatorname{Re}(\mu+\nu)>-1$, then it follows from Gradshteyn ([7, p. 707) that

$$
\begin{aligned}
\int_{0}^{1} x^{\mu} J_{\nu}(\omega x) d x=\frac{2^{\mu} \Gamma\left(\frac{\mu+\nu+1}{2}\right)}{\omega^{\mu+1} \Gamma\left(\frac{\nu-\mu+1}{2}\right)} & +\omega^{-\mu}\left\{(\mu+\nu-1) J_{\nu}(\omega) s_{\mu-1, \nu-1}^{(2)}(\omega)\right. \\
& \left.-J_{\nu-1}(\omega) s_{\mu, \nu}^{(2)}(\omega)\right\}
\end{aligned}
$$

where $s_{\mu, \nu}^{(2)}(z)$ denotes the second kind of Lommel function. According to the preceding conditions $\operatorname{Re}(m)>-\frac{1}{r+1}$ and $y_{0}>0$, we can deduce that $\operatorname{Re}(m)+\frac{k+1}{r+1}-1>-1$ and $\omega y_{0}^{r+1}>0$. Therefore, we can obtain the moments for $J_{m}\left(\omega t^{r+1}\right)$ immediately from (2.5):

$$
\begin{aligned}
I\left[t^{k}\right]= & \int_{0}^{y_{0}} t^{k} J_{m}\left(\omega t^{r+1}\right) d t \\
= & \frac{y_{0}^{k+1}}{r+1} \int_{0}^{1} x^{\frac{k+1}{r+1}-1} J_{m}\left(\omega y_{0}^{r+1} x\right) d x \\
= & \frac{y_{0}^{k+1}}{r+1}\left[\frac{2^{\frac{k+1}{r+1}-1} \Gamma\left(\frac{m+\frac{k+1}{r+1}}{2}\right)}{\left(\omega y_{0}^{r+1}\right)^{\frac{k+1}{r+1}} \Gamma\left(\frac{m-\frac{k+1}{r+1}+2}{2}\right)}+\left(\omega y_{0}^{r+1}\right)^{-\frac{k+1}{r+1}+1} .\right. \\
& \left\{\left(\frac{k+1}{r+1}+m-2\right) J_{m}\left(\omega y_{0}^{r+1}\right) s_{\frac{k+1}{r+1}-2, m-1}^{(2)}\left(\omega y_{0}^{r+1}\right)\right. \\
& \left.\left.-J_{m-1}\left(\omega y_{0}^{r+1}\right) s_{\frac{k+1}{r+1}-1, m}^{(2)}\left(\omega y_{0}^{r+1}\right)\right\}\right] .
\end{aligned}
$$

For large values of $z, s_{\mu, \nu}^{(2)}(z)$ admits the following asymptotic expansion (24], p. 351):

$$
\begin{aligned}
& s_{\mu, \nu}^{(2)}(z) \\
& =z^{\mu-1}\left[1-\frac{(\mu-1)^{2}-\nu^{2}}{z^{2}}+\frac{\left\{(\mu-1)^{2}-\nu^{2}\right\}\left\{(\mu-3)^{2}-\nu^{2}\right\}}{z^{4}}-\cdots\right] \\
& =z^{\mu-1}\left[1-\frac{(\mu-1)^{2}-\nu^{2}}{z^{2}}+\cdots\right. \\
& \left.+(-1)^{p} \frac{\left\{(\mu-1)^{2}-\nu^{2}\right\} \cdots\left\{(\mu-2 p+1)^{2}-\nu^{2}\right\}}{z^{2 p}}\right]+O\left(z^{\mu-2 p-2}\right) .
\end{aligned}
$$

Therefore, $s_{\mu, \nu}^{(2)}(z)$ can be efficiently approximated by truncating (2.7) once $\omega$ is large. Furthermore, if either of the numbers $\mu \pm \nu$ is an odd positive integer, then the Lommel function $s_{\mu, \nu}^{(2)}(z)$ has a finite representation of (2.7) $(24$, p. 347).

- For $S\left(\omega t^{r+1}\right)=A i\left(-\omega t^{r+1}\right)$ : By

$$
A i(-x)=\frac{1}{3} \sqrt{x}\left[J_{-\frac{1}{3}}\left(\frac{2}{3} x^{\frac{3}{2}}\right)+J_{\frac{1}{3}}\left(\frac{2}{3} x^{\frac{3}{2}}\right)\right]([1], \text { p. 447) }
$$

the moment $I\left[t^{k}\right]=\int_{0}^{1} t^{k} A i\left(-\omega t^{r+1}\right) d t$ can be represented by 


$$
\begin{aligned}
& I\left[t^{k}\right]=\int_{0}^{y_{0}} t^{k} A i\left(-\omega t^{r+1}\right) d t=\frac{y_{0}^{r+1}}{r+1} \int_{0}^{1} t^{\frac{k-r}{r+1}} A i\left(-\omega y_{0}^{r+1} t\right) d t \\
&= \frac{\sqrt{\omega} y_{0}^{k+1+\frac{r+1}{2}}}{9(r+1)}\left[\frac{2^{\frac{2 k-2 r}{3 r+3}} \Gamma\left(\frac{k+1}{3 r+3}\right)}{\left(\frac{2}{3}\left(\omega y_{0}^{r+1}\right)^{\frac{3}{2}}\right)^{\frac{2 k+r+3}{3 r+3}} \Gamma\left(\frac{2 r-k+1}{3 r+3}\right)}+\frac{2^{\frac{2 k-2 r}{3 r+3}} \Gamma\left(\frac{k+r+2}{3 r+3}\right)}{\left(\frac{2}{3}\left(\omega y_{0}^{r+1}\right)^{\frac{3}{2}}\right)^{\frac{2 k+r+3}{3 r+3}} \Gamma\left(\frac{3 r-k+2}{3 r+3}\right)}\right. \\
&+\left(\frac{2}{3}\left(\omega y_{0}^{r+1}\right)^{\frac{3}{2}}\right)^{-\frac{2 k-2 r}{3 r+3}}\left\{\left(\frac{2 k-6 r-4}{3 r+3}\right) J_{-\frac{1}{3}}\left(\frac{2}{3}\left(\omega y_{0}^{r+1}\right)^{\frac{3}{2}}\right) s_{\frac{2 k-5 r-3}{3 r+3},-\frac{4}{3}}^{(2)}\left(\frac{2}{3}\left(\omega y_{0}^{r+1}\right)^{\frac{3}{2}}\right)\right. \\
& \quad-J_{-\frac{4}{3}}\left(\frac{2}{3}\left(\omega y_{0}^{r+1}\right)^{\frac{3}{2}}\right) s_{\frac{2 k-2 r}{3 r+3},-\frac{1}{3}}^{(2)}\left(\frac{2}{3}\left(\omega y_{0}^{r+1}\right)^{\frac{3}{2}}\right) \\
&+\left(\frac{2 k-4 r-2}{3 r+3}\right) J_{\frac{1}{3}}\left(\frac{2}{3}\left(\omega y_{0}^{r+1}\right)^{\frac{3}{2}}\right) s_{\frac{2 k-5 r-3}{3 r+3},-\frac{2}{3}}^{(2)}\left(\frac{2}{3}\left(\omega y_{0}^{r+1}\right)^{\frac{3}{2}}\right) \\
&\left.\left.\quad-J_{-\frac{2}{3}}\left(\frac{2}{3}\left(\omega y_{0}^{r+1}\right)^{\frac{3}{2}}\right) s_{\frac{2 k-2 r}{3 r+3}, \frac{1}{3}}^{(2)}\left(\frac{2}{3}\left(\omega y_{0}^{r+1}\right)^{\frac{3}{2}}\right)\right\}\right] .
\end{aligned}
$$

\section{Numerical ANALYSis FOR THE FILON-TYPE METHOD} FOR THE BESSEL TRANSFORM

In this section, we consider the Filon-type method (2.4) for

$$
I[f]=\int_{0}^{1} f(x) J_{m}(\omega g(x)) d x,
$$

where $f(x)$ and $g(x)$ are smooth functions and $g(x)$ satisfies (1.7) with $g^{(r+1)}(0)>0$. In the case of $g^{(r+1)}(0)<0$, by using $J_{m}(x)=e^{-i m \pi} J_{m}(-x)$ ([1], p. 361]), the integral (3.1) can be transformed into $e^{-i m \pi} \int_{0}^{1} f(x) J_{m}(-\omega g(x)) d x$ with $-g^{(r+1)}(0)>$ 0 .

Lemma 3.1. Suppose that $\alpha$ is a real number with $0 \leq \alpha \leq r$. Then for every function $w(t) \in C^{1}\left[0, y_{0}\right]$, we have

$$
\left|\int_{0}^{y_{0}} w(t) t^{\alpha} J_{m}\left(\omega t^{r+1}\right) d t\right| \leq \frac{C}{\omega^{(\alpha+1) /(r+1)}}\left(\left|w\left(y_{0}\right)\right|+\int_{0}^{y_{0}}\left|w^{\prime}(t)\right| d t\right)
$$

for some constants $C$ independent of $w(t)$ and $\omega$.

Proof. We first show that for all $x \in\left[0, y_{0}\right]$,

$$
\left|\int_{0}^{x} t^{\alpha} J_{m}\left(\omega t^{r+1}\right) d t\right| \leq C \omega^{-(\alpha+1) /(r+1)}
$$

for some constant $C$ independent of $x, \omega$ and $w(t)$. Let $u=\omega t^{r+1}$. Then, the integral in (3.3) is expressed by

$$
\int_{0}^{x} t^{\alpha} J_{m}\left(\omega t^{r+1}\right) d t=\frac{\omega^{-(\alpha+1) /(r+1)}}{r+1} \int_{0}^{\omega x^{r+1}} u^{\frac{\alpha-r}{r+1}} J_{m}(u) d u .
$$

Therefore, it is trivial to prove (3.3) if the integral in the right-hand side of (3.4) is uniformly bounded on $x$ and $\omega$. Recalling from the asymptotic expansion of $s_{\mu, \nu}^{(2)}(u)$ in (2.7), for large $u$ one can obtain

$$
s_{\frac{\alpha-r}{r+1}-1, m-1}^{(2)}(u)=O\left(u^{\frac{\alpha-r}{r+1}-2}\right), \quad s_{\frac{\alpha-r}{r+1}, m}^{(2)}(u)=O\left(u^{\frac{\alpha-r}{r+1}-1}\right) .
$$


This, together with the following asymptotic expansion ([1], p. 364; 22], p. 338)

$$
J_{\nu}(u)=\left(\frac{2}{\pi}\right)^{\frac{1}{2}} u^{-\frac{1}{2}} \cos \left(u-\frac{\nu \pi}{2}-\frac{\pi}{4}\right)+O\left(u^{-\frac{3}{2}}\right), \quad u \gg 1
$$

and with (2.6), deduces that $\int_{0}^{+\infty} u^{\frac{\alpha-r}{r+1}} J_{m}(u) d u$ is convergent, and then the function $G(z)=\int_{0}^{z} u^{\frac{\alpha-r}{r+1}} J_{m}(u) d u$ is continuous and uniformly bounded in $[0,+\infty)$, which implies that $\int_{0}^{\omega x^{r+1}} u^{\frac{\alpha-r}{r+1}} J_{m}(u) d u$ is uniformly bounded on $\left[0, y_{0}\right]$.

Expression (3.2) is proved by writing $\int_{0}^{y_{0}} w(t) t^{\alpha} J_{m}\left(\omega t^{r+1}\right) d t$ as $\int_{0}^{y_{0}} w(t) F^{\prime}(t) d t$, with

$$
F(t)=\int_{0}^{t} u^{\alpha} J_{m}\left(\omega u^{r+1}\right) d u
$$

Integrating by parts one derives

$$
\int_{0}^{y_{0}} w(t) t^{\alpha} J_{m}\left(w t^{r+1}\right) d t=w\left(y_{0}\right) F\left(y_{0}\right)+\int_{0}^{y_{0}} w^{\prime}(t) F(t) d t,
$$

which together with (3.3) gives the desired result.

Example 3.1. Let us consider the asymptotic behaviour of $I\left[t^{k}\right]=\int_{0}^{1} t^{k} J_{0}\left(\omega t^{3}\right) d t$ for large $\omega$ (see Figure 1).

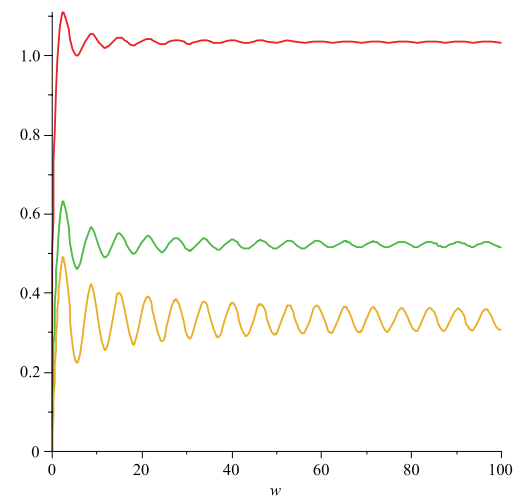

Figure 1. The absolute values of the moment $I\left[t^{k}\right]=$ $\int_{0}^{1} t^{k} J_{0}\left(\omega t^{3}\right) d t$ scaled by $\omega^{(k+1) / 3}$ for $k=0,1,2$ (the top, middle and bottom, respectively) and $0 \leq \omega \leq 100$.

The asymptotic expansion (1.3) of the highly oscillatory integral $\int_{0}^{1} f(x) e^{i \omega g(x)} d x$ provides an invaluable tool in numerical analysis [11. For the integral $\int_{0}^{y_{0}} f(x) J_{m}(\omega x) d x$, the asymptotic expansion can be obtained from the following lemma. 
Lemma 3.2. For every smooth $f$ it is true that

$$
\begin{aligned}
\int_{0}^{y_{0}} f(x) J_{m}(\omega x) d x & \sim \sum_{k=0}^{+\infty} \frac{(-1)^{k}}{\omega^{k}} \sigma_{k}[f](0) \int_{0}^{y_{0}} J_{m+k}(\omega x) d x \\
& -\sum_{k=1}^{+\infty} \frac{(-1)^{k}}{\omega^{k}}\left\{\sigma_{k-1}[f]\left(y_{0}\right)-\sigma_{k-1}[f](0)\right\} J_{m+k}\left(\omega y_{0}\right),
\end{aligned}
$$

where $\int_{0}^{y_{0}} J_{m+k}(\omega x) d x$ can be computed explicitly by (2.6) and

$$
\begin{gathered}
\sigma_{0}[f](x)=f(x), \\
\sigma_{k+1}[f](x)=\frac{x \sigma_{k}[f]^{\prime}(x)-(m+k+1)\left(\sigma_{k}[f](x)-\sigma_{k}[f](0)\right)}{x}, \quad k \geq 0 .
\end{gathered}
$$

Proof. Following Iserles and Nørsett [1], we prove by induction on $s$ the identity

$$
\begin{array}{rl}
\int_{0}^{y_{0}} & f(x) J_{m}(\omega x) d x \\
= & \sum_{k=0}^{s-1} \frac{(-1)^{k}}{\omega^{k}} \sigma_{k}[f](0) \int_{0}^{y_{0}} J_{m+k}(\omega x) d x \\
& -\sum_{k=1}^{s} \frac{(-1)^{k}}{\omega^{k}}\left\{\sigma_{k-1}[f]\left(y_{0}\right)-\sigma_{k-1}[f](0)\right\} J_{m+k}\left(\omega y_{0}\right) \\
& +\frac{(-1)^{s}}{\omega^{s}} \int_{0}^{y_{0}} \sigma_{s}[f](x) J_{m+s}(\omega x) d x .
\end{array}
$$

Suppose that $s=1$. From $\frac{d}{d x}\left[x^{m+1} J_{m+1}(x)\right]=x^{m+1} J_{m}(x)$ ([1], p. 361]), we have

$$
\begin{array}{rl}
\int_{0}^{y_{0}} & f(x) J_{m}(\omega x) d x \\
= & \sigma_{0}[f](0) \int_{0}^{y_{0}} J_{m}(\omega x) d x+\frac{1}{\omega} \int_{0}^{y_{0}} \frac{\sigma_{0}[f](x)-\sigma_{0}[f](0)}{x^{m+1}} d\left[x^{m+1} J_{m+1}(\omega x)\right] \\
= & \sigma_{0}[f](0) \int_{0}^{y_{0}} J_{m}(\omega x) d x+\frac{1}{\omega}\left(\sigma_{0}[f]\left(y_{0}\right)-\sigma_{0}[f](0)\right) J_{m+1}\left(\omega y_{0}\right) \\
& -\frac{1}{\omega} \int_{0}^{y_{0}} \frac{x \sigma_{0}[f]^{\prime}(x)-(m+1)\left(\sigma_{0}[f](x)-\sigma_{0}[f](0)\right)}{x} J_{m+1}(\omega x) d x \\
= & \sigma_{0}[f](0) \int_{0}^{y_{0}} J_{m}(\omega x) d x+\frac{1}{\omega}\left(\sigma_{0}[f]\left(y_{0}\right)-\sigma_{0}[f](0)\right) J_{m+1}\left(\omega y_{0}\right) \\
& -\frac{1}{\omega} \int_{0}^{y_{0}} \sigma_{1}[f](x) J_{m+1}(\omega x) d x
\end{array}
$$


So it is true for $s=1$. For $s \geq 2$, integration by parts on the right yields

$$
\begin{aligned}
\frac{(-1)^{s}}{\omega^{s}} & \int_{0}^{y_{0}} \sigma_{s}[f](x) J_{m+s}(\omega x) d x \\
= & \frac{(-1)^{s} \sigma_{s}[f](0)}{\omega^{s}} \int_{0}^{y_{0}} J_{m+s}(\omega x) d x \\
& +\frac{(-1)^{s}}{\omega^{s+1}} \int_{0}^{y_{0}} \frac{\sigma_{s}[f](x)-\sigma_{s}[f](0)}{x^{m+s+1}} d\left[x^{m+s+1} J_{m+s+1}(\omega x)\right] \\
= & \frac{(-1)^{s} \sigma_{s}[f](0)}{\omega^{s}} \int_{0}^{y_{0}} J_{m+s}(\omega x) d x \\
& +\left.\frac{(-1)^{s}\left(\sigma_{s}[f](x)-\sigma_{s}[f](0)\right)}{\omega^{s+1}} J_{m+s+1}(\omega x)\right|_{0} ^{y_{0}} \\
& +\frac{(-1)^{s+1}}{\omega^{s+1}} \int_{0}^{y_{0}} \sigma_{s+1}[f](x) J_{m+s+1}(\omega x) d x
\end{aligned}
$$

This proves (3.8). Letting $s \rightarrow \infty$ yields the asymptotic expansion (3.7).

The following asymptotic quadrature for $\int_{0}^{y_{0}} f(x) J_{m}(\omega x) d x$, defined by

$$
\begin{aligned}
Q_{s}^{A}[f]= & \sum_{k=0}^{s-1} \frac{(-1)^{k}}{\omega^{k}} \sigma_{k}[f](0) \int_{0}^{y_{0}} J_{m+k}(\omega x) d x \\
& -\sum_{k=1}^{s} \frac{(-1)^{k}}{\omega^{k}}\left\{\sigma_{k-1}[f]\left(y_{0}\right)-\sigma_{k-1}[f](0)\right\} J_{m+k}\left(\omega y_{0}\right),
\end{aligned}
$$

a truncation of the asymptotic expansion (3.7), is efficient for large values of $\omega$.

Theorem 3.1. For every smooth $f$ it is true that

$$
Q_{s}^{A}[f]-\int_{0}^{y_{0}} f(x) J_{m}(\omega x) d x=O\left(\frac{1}{\omega^{s+1}}\right), \quad \omega \gg 1 .
$$

Proof. This follows directly from Lemma 3.1 and (3.8).

Throughout the rest of the paper, we evaluate $s_{\mu, \nu}^{(2)}(z)$ by truncating after the first 10 terms of (2.7). The exact values of the integrals in the numerical examples are computed by MAPLE 11 with 100-digit arithmetic; they are also tested by using the code of Clenshaw-Curtis methods presented by Trefethen in [23] with 32-digit or 64-digit arithmetic, respectively.

Example 3.2. Let us consider the asymptotic quadrature (3.9) for approximating

$$
\int_{0}^{1} \cos (x) J_{0}(\omega x) d x \text { (see Figure 2). }
$$



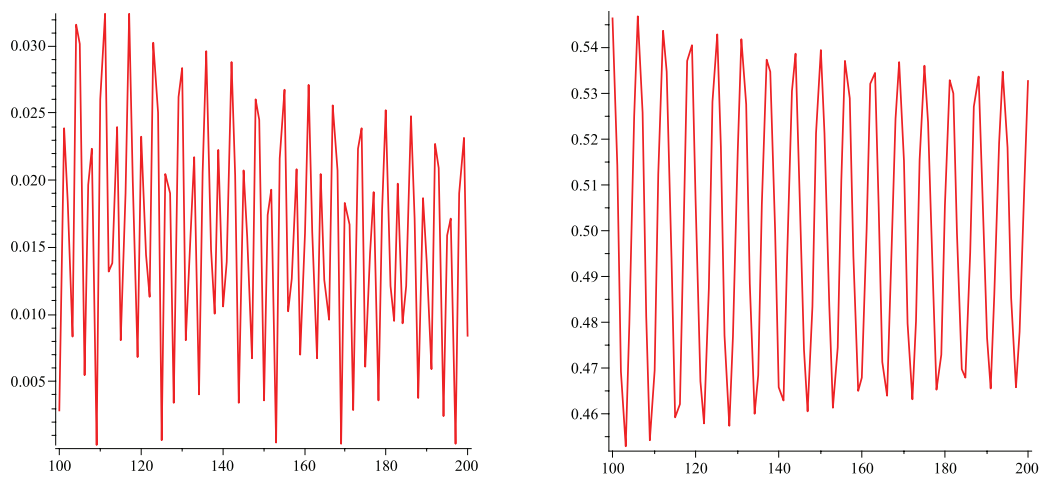

Figure 2. The absolute error for $Q_{1}^{A}[\cos (x)]$ (the left) and $Q_{2}^{A}[\cos (x)]$ (the right) scaled by $\omega^{2}$ and $\omega^{3}$, respectively, for $\int_{0}^{1} \cos (x) J_{0}(\omega x) d x$ and $100 \leq \omega \leq 200$.

Applying the asymptotic expansion (3.7), we give the error analysis of the Filontype method (2.4) for the Bessel transform in (1.6).

Theorem 3.2. The Filon-type method defined by (2.4) for

$$
I[f]=\int_{0}^{1} f(x) J_{m}(\omega g(x)) d x
$$

satisfies

$$
I[f]-Q_{s}^{F}[f]=O\left(\frac{1}{\omega^{s+\left(k_{0}+1\right) /(r+1)}}\right) .
$$

Proof. Let $h(t)=\tilde{f}(t)-p(t)$ and $x=t^{r+1}$. Then from (2.2) and (2.4) the error can be written as

$$
\begin{aligned}
I[f]- & Q_{s}^{F}[f]=(r+1) \int_{0}^{y_{0}} h(t) J_{m}\left(\omega t^{r+1}\right) d t \\
& =\int_{0}^{y_{0}^{r+1}} \frac{h\left(x^{1 /(r+1)}\right)}{x^{r /(r+1)}} J_{m}(\omega x) d x \\
& =\int_{0}^{y_{0}^{r+1}} \psi(x) J_{m}(\omega x) d x,
\end{aligned}
$$

where $\psi(x)=\frac{h\left(x^{1 /(r+1)}\right)}{x^{r /(r+1)}}$. From Maclaurin's expansion of

$$
h(t)=\frac{\widetilde{f}^{\left(s(r+1)+k_{0}\right)}(\xi)-p^{\left(s(r+1)+k_{0}\right)}(\xi)}{\left(s(r+1)+k_{0}\right) !} t^{s(r+1)+k_{0}} \text { for some } \xi \in[0, t],
$$

we have

$$
\left\{\begin{array}{r}
\psi(x)=\frac{\tilde{f}^{\left(s(r+1)+k_{0}\right)}(\xi)-p^{\left(s(r+1)+k_{0}\right)}(\xi)}{\left(s(r+1)+k_{0}\right) !} x^{s+\frac{k_{0}-r}{r+1}} \\
\psi(0)=\psi^{\prime}(0)=\cdots=\psi^{(s-1)}(0)=0, \psi\left(y_{0}^{r+1}\right) \\
=\psi^{\prime}\left(y_{0}^{r+1}\right)=\cdots=\psi^{(s-1)}\left(y_{0}^{r+1}\right)=0 .
\end{array}\right.
$$


It is not difficult from (3.12) and the definitions of $\sigma_{k}[\psi](x)$ to verify that

$$
\sigma_{1}[\psi](x)=O\left(x^{s-1+\frac{k_{0}-r}{r+1}}\right), \ldots, \sigma_{s}[\psi](x)=O\left(x^{\frac{k_{0}-r}{r+1}}\right)
$$

and

Therefore

$$
\sigma_{k}[\psi](0)=\sigma_{k}[\psi]\left(y_{0}^{r+1}\right)=0, \quad k=0, \ldots, s-1 .
$$

$$
\begin{gathered}
\sigma_{1}[\psi]\left(t^{r+1}\right)=O\left(t^{(s-1)(r+1)+k_{0}-r}\right), \ldots, \sigma_{s}[\psi]\left(t^{r+1}\right)=O\left(t^{k_{0}-r}\right), \\
\sigma_{s}[\psi]\left(t^{r+1}\right) t^{r}=O\left(t^{k_{0}}\right) .
\end{gathered}
$$

This, together with (3.8) and Lemma 3.1, implies

$$
\begin{aligned}
I[f]-Q_{s}^{F}[f] & =\frac{(-1)^{s}}{\omega^{s}} \int_{0}^{y_{0}^{r+1}} \sigma_{s}[\psi](x) J_{m+s}(\omega x) d x \\
& =\frac{(-1)^{s}(r+1)}{\omega^{s}} \int_{0}^{y_{0}} \sigma_{s}[\psi]\left(t^{r+1}\right) t^{r} J_{m+s}\left(\omega t^{r+1}\right) d t \\
& =O\left(\frac{1}{\omega^{s+\left(k_{0}+1\right) /(r+1)}}\right) .
\end{aligned}
$$

Example 3.3. We now demonstrate the application of the Filon-type method to several numerical examples. The first is the computation of $\int_{0}^{1} e^{x} J_{0}(\omega(1-$ $\cos (x))) d x$. It is evident that the Filon-type method is applicable. We compare two
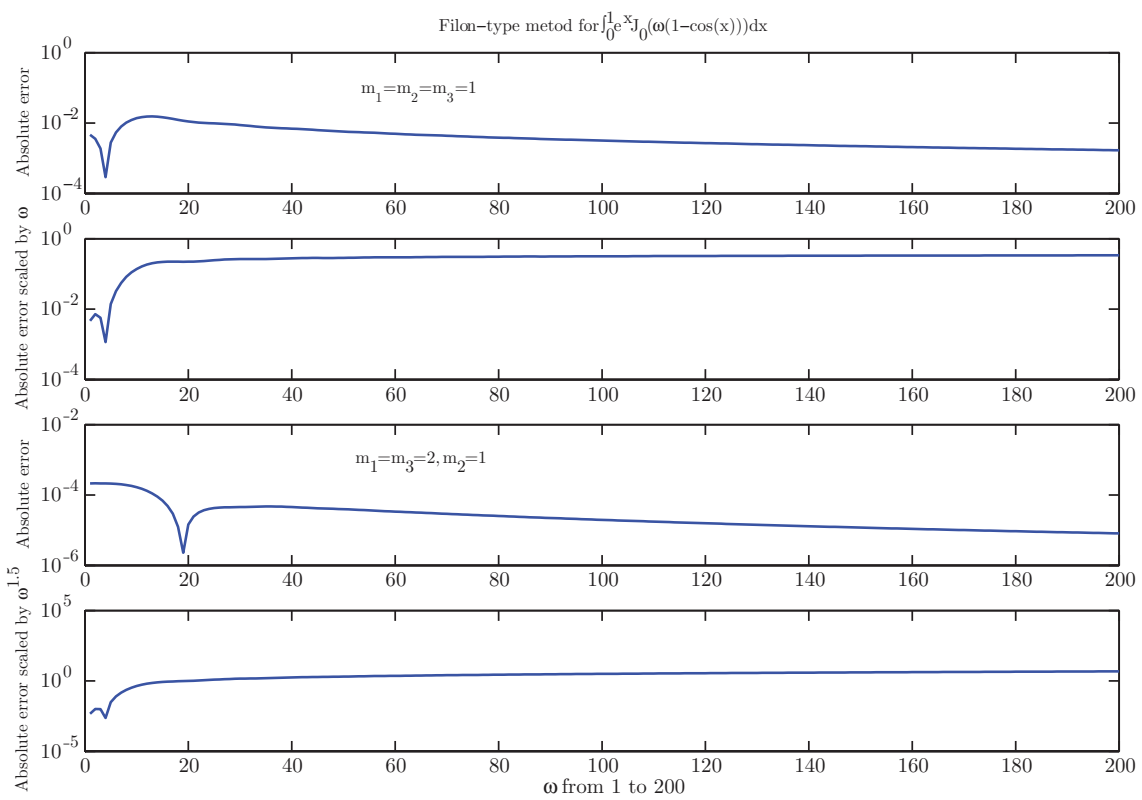

Figure 3. The error (the top), error scaled by $\omega$ (the second), the error (the third), and error scaled by $\omega^{1.5}$ (bottom) with the same nodes $\left\{x_{j}=\frac{\left(\cos \left(\frac{(n-j) \pi}{2}\right)+1\right)}{2}, j=1,2,3\right\}$, and multiplicities all one and $\{2,1,2\}$, respectively, for $\int_{0}^{1} e^{x} J_{0}(\omega(1-\cos (x))) d x$. 
Filon-type methods with the same three nodes and multiplicities all 1 and $\{2,1,2\}$, respectively. As seen in Figure 3, the first two figures illustrate the errors (the top) and errors scaled by $\omega$ (the second) with nodes $\left\{x_{j}=\frac{\left(\cos \left(\frac{(3-j) \pi}{2}\right)+1\right)}{2}, j=1,2,3\right\}$ and multiplicities all 1 . The bottom two figures illustrate the errors and errors scaled by $\omega^{1.5}$ with the same nodes and multiplicities $\{2,1,2\}$, respectively.

The other two examples are $I\left[\frac{1}{1+x}\right]=\int_{0}^{1} \frac{1}{1+x} J_{1}\left(\omega\left(e^{x}-x-1\right)\right) d x$ and $I\left[e^{x}\right]=$ $\int_{0}^{1} e^{x} J_{2}(\omega(x-\sin (x))) d x$, which seem more complicated to handle. Tables 1 and 2 illustrate the relative errors in $v$-points approximation with multiplicities all 1 . From these two tables, we can see that the Filon-type method exhibits the fast convergence as $v$ increases. Tables 3 and 4 illustrate that as the multiplicities increase at the end points, the approximate accuracy can be enhanced.

TABLE 1. Relative errors in $v$-points approximation to $I\left[\frac{1}{1+x}\right]=$ $\int_{0}^{1} \frac{1}{1+x} J_{1}\left(\omega\left(e^{x}-x-1\right)\right) d x$ with multiplicities all one.

\begin{tabular}{|c|c|c|c|}
\hline$\omega$ & $v=4$ & $v=8$ & $v=12$ \\
\hline 200 & 0.0046263225 & 0.0000085084 & $1.427796502 E-8$ \\
\hline 500 & 0.0039959151 & 0.0000036176 & $2.983185440 E-9$ \\
\hline 1000 & 0.0032365958 & $2.200974762 E-7$ & $8.707204300 E-9$ \\
\hline 2000 & 0.0025035221 & 0.0000023876 & $6.252544276 E-9$ \\
\hline
\end{tabular}

TABLE 2. Relative errors in $v$-points approximation to $I\left[e^{x}\right]=$ $\int_{0}^{1} e^{x} J_{2}(\omega(x-\sin (x))) d x$ with multiplicities all one.

\begin{tabular}{|c|c|c|c|}
\hline$\omega$ & $v=4$ & $v=8$ & $v=12$ \\
\hline 200 & 0.001656537 & $4.586916397 E-8$ & $7.026658595 E-14$ \\
\hline 500 & 0.0005901539 & $1.574877810 E-7$ & $3.537131115 E-12$ \\
\hline 1000 & 0.0002677535 & $1.160995982 E-7$ & $9.436098047 E-12$ \\
\hline 2000 & 0.0009114874 & $7.731021307 E-9$ & $4.422931661 E-12$ \\
\hline
\end{tabular}

TABLE 3. Relative errors in $v$-points approximation to $I\left[\frac{1}{1+x}\right]=$ $\int_{0}^{1} \frac{1}{1+x} J_{1}\left(\omega\left(e^{x}-x-1\right)\right) d x$ with $m_{1}, m_{v}=2$ and the others all one.

\begin{tabular}{|c|c|c|c|}
\hline$\omega$ & $v=4$ & $v=8$ & $v=12$ \\
\hline 200 & 0.0003147118 & $6.597628622 E-7$ & $1.309191824 E-9$ \\
\hline 500 & 0.0001758496 & $1.734538903 E-7$ & $1.591356020 E-10$ \\
\hline 1000 & 0.0001015467 & $9.101387022 E-9$ & $3.248618020 E-10$ \\
\hline 2000 & 0.0000558027 & $5.724619643 E-8$ & $1.581881723 E-10$ \\
\hline
\end{tabular}


TABLE 4. Relative errors in $v$-points approximation to $I\left[e^{x}\right]=$ $\int_{0}^{1} e^{x} J_{2}(\omega(x-\sin (x))) d x$ with $m_{1}, m_{v}=2$ and the others all one.

\begin{tabular}{|c|c|c|c|}
\hline$\omega$ & $v=4$ & $v=8$ & $v=12$ \\
\hline 200 & 0.0000618306 & $1.673703661 E-9$ & $2.112253035 E-14$ \\
\hline 500 & 0.0000196664 & $4.445163366 E-9$ & $1.098853818 E-13$ \\
\hline 1000 & 0.0000067988 & $2.896855435 E-9$ & $2.289120828 E-13$ \\
\hline 2000 & 0.0000208972 & $9.401439940 E-11$ & $9.561286297 E-14$ \\
\hline
\end{tabular}

\section{An applichtion to the Airy transform}

In this section, we consider the Filon-type method for the integral

$$
I[f]=\int_{0}^{1} f(x) A i(-\omega g(x)) d x
$$

where $f(x)$ and $g(x)$ are smooth functions and $g(x)$ satisfies $(1.7)$ with $g^{(r+1)}(0)>0$. Using the transformation $t^{r+1}=g(x)$, (4.1) can be transformed into

$$
I[f]=(r+1) \int_{0}^{y_{0}} \widetilde{f}(t) A i\left(-\omega t^{r+1}\right) d t,
$$

where $\tilde{f}(t)$ is a smooth function.

Assume that $s$ is a positive integer and that $\left\{m_{k}\right\}_{1}^{v}$ is a set of multiplicities associated with the node points $0=d_{1}<d_{2}<\cdots<d_{v}=y_{0}$ such that $m_{1} \geq$ floor $\left(\frac{(3 s-1)(r+1)+k_{0}}{2}\right)\left(0 \leq k_{0}<3 r+3\right)$ and $m_{v} \geq s$, where floor $(x)$ rounds the elements of $x$ to the nearest integers towards minus infinity. Suppose that $p(t)$ is the Hermite interpolating polynomial defined by $(2.3)$ and $h(t)=\widetilde{f}(t)-p(t)$.

Based on

$$
A i(-x)=\frac{1}{3} \sqrt{x}\left[J_{-\frac{1}{3}}\left(\frac{2}{3} x^{\frac{3}{2}}\right)+J_{\frac{1}{3}}\left(\frac{2}{3} x^{\frac{3}{2}}\right)\right],
$$

the error can be rewritten by $t=u^{2}$ as

$$
\begin{aligned}
I[f]-Q_{s}^{F}[f]=\frac{2(r+1) \sqrt{\omega}}{3} \int_{0}^{\sqrt{y_{0}}} h\left(u^{2}\right) u^{r+2} & {\left[J_{-\frac{1}{3}}\left(\frac{2}{3} \omega^{\frac{3}{2}} u^{3 r+3}\right)\right.} \\
& \left.+J_{\frac{1}{3}}\left(\frac{2}{3} \omega^{\frac{3}{2}} u^{3 r+3}\right)\right] d u .
\end{aligned}
$$

Theorem 4.1. The Filon-type method defined by (2.4) with

$$
m_{1} \geq \text { floor }\left(\frac{(3 s-1)(r+1)+k_{0}}{2}\right), \quad m_{v} \geq s,
$$

for $I[f]=\int_{0}^{1} f(x) A i(-\omega g(x)) d x$ satisfies

$$
I[f]-Q_{s}^{F}[f]=O\left(\frac{1}{\omega^{\frac{3 s-1}{2}+\frac{k_{0}+1}{2(r+1)}}}\right) .
$$

Proof. From (4.3), we see that $u=0$ and $u=\sqrt{y_{0}}$ are two roots of $h\left(u^{2}\right) u^{r+2}=0$ with multiplicities $\widetilde{m_{1}} \geq 3 s(r+1)+k_{0}$ and $\widetilde{m_{v}} \geq s$, respectively. Therefore, from 
Theorem 3.2, we have

$$
I[f]-Q_{s}^{F}[f]=O\left(\frac{\omega^{\frac{1}{2}}}{\left(\omega^{\frac{3}{2}}\right)^{\frac{3 s(r+1)+k_{0}+1}{3(r+1)}}}\right)=O\left(\frac{1}{\omega^{\frac{3 s-1}{2}+\frac{k_{0}+1}{2(r+1)}}}\right) .
$$

Example 4.1. As an illustration of the effectiveness of the Filon-type method, we consider the Filon-type method $Q_{s}^{F}\left[\frac{1}{1+x}\right](s=1,2)$ in the case $r=0$ for approximating

$$
I\left[\frac{1}{1+x}\right]=\int_{0}^{1} \frac{1}{1+x} A i(-\omega x) d x(\text { see Figure } 4) .
$$

Filon-type method $\mathrm{Q}_{\mathrm{S}}^{\mathrm{F}}[1 /(1+\mathrm{x})]$ for $\int_{0}^{1} 1 /(1+\mathrm{x}) \mathrm{Ai}(-\omega \mathrm{x}) \mathrm{dx}(\mathrm{s}=1,2)$
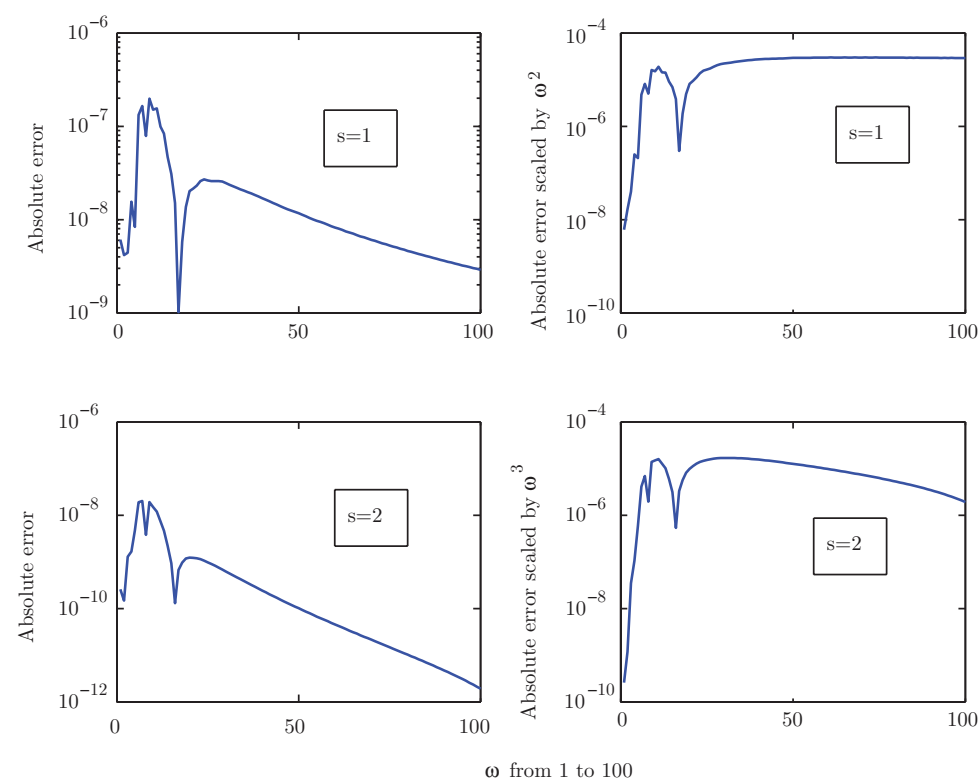

Figure 4. The absolute error and the absolute error scaled by $\omega^{2}$ and $\omega^{3}$, respectively, for the Filon-type method with $m_{1}=\cdots=$ $m_{8}=1$ (the first row), and $m_{1}=m_{8}=2, m_{2}=\cdots=m_{7}=1$ (the second row) at shifted Chebyshev points $\left\{x_{j}=\frac{\left(\cos \left(\frac{(8-j) \pi}{7}\right)+1\right)}{2}, j=\right.$ $1,2, \ldots, 8\}$, for $I[f]=\int_{0}^{1} \frac{1}{1+x} A i(-\omega x) d x$.

Remark 1. The asymptotic order on $\omega$ of the error bound (4.4) of the Filon-type method (2.4) for the Airy transform is sharp. For example, let us consider

$$
\int_{0}^{1}\left(x^{3}+2 x+1+x^{2}(1+\cos (\pi x))\right) A i(-\omega x) d x .
$$

The polynomial $x^{3}+2 x+1$ is the Hermite interpolating polynomial of $x^{3}+2 x+1+$ $x^{2}(1+\cos (\pi x))$ at 0 and 1 with multiplicities all 2 . So the error for the Filon-type 
method can be written as

$$
I[f]-Q_{2}^{F}[f]=\int_{0}^{1} x^{2}(1+\cos (\pi x)) A i(-\omega x) d x .
$$

Since $[A i(x)]^{\prime \prime}=x A i(x)\left(\left[1\right.\right.$, p. 446), it follows that $\omega^{3} x A i(-\omega x)=-[A i(-\omega x)]^{\prime \prime}$. Integrating by parts twice we get for $\omega \gg 1$,

$$
\begin{aligned}
I[f]-Q_{2}^{F}[f] & =-\frac{1}{\omega^{3}} \int_{0}^{1} x(1+\cos (\pi x))[A i(-\omega x)]^{\prime \prime} d x \\
& =-\frac{2}{\omega^{3}} A i(0)+\frac{\pi}{\omega^{3}} \int_{0}^{1}(2 \sin (\pi x)+\pi x \cos (\pi x)) A i(-\omega x) d x \\
& =O\left(\frac{1}{\omega^{3}}\right)
\end{aligned}
$$

since $A i(0)=3^{-2 / 3} / \Gamma\left(\frac{2}{3}\right) \neq 0$ and

$$
\begin{aligned}
& \frac{\pi}{\omega^{3}} \int_{0}^{1}(2 \sin (\pi x)+\pi x \cos (\pi x)) A i(-\omega x) d x \\
= & \frac{\pi}{3 \omega^{3}} \int_{0}^{1}(2 \sin (\pi x)+\pi x \cos (\pi x)) \sqrt{x}\left[J_{-\frac{1}{3}}\left(\frac{2}{3} x^{\frac{3}{2}}\right)+J_{\frac{1}{3}}\left(\frac{2}{3} x^{\frac{3}{2}}\right)\right] \\
= & O\left(\frac{1}{\omega^{4}}\right) \text { (Lemma 3.1). }
\end{aligned}
$$

\section{ACKNOWLEDGEMENT}

The first author would like to express his sincere gratitude to Prof. A. Iserles and Prof. S. P. Nørsett et al. for their helpful discussions during the Workshop "Highly Oscillatory Problems: Computation, Theory and Applications" at the Isaac Newton Institute for Mathematical Sciences, University of Cambridge in 2007. The authors are grateful to Prof. Xiaojun Chen, Dr. Andbach An, the associated editor and the two anonymous referees for their useful comments and helpful suggestions for the improvement of this paper.

\section{REFERENCES}

[1] M. Abramowitz and I. A. Stegun, Handbook of Mathematical Functions: with Formulas, Graphs, and Mathematical Tables, Dover Publications, Inc., New York, 1965. MR.1225604 (94b:00012)

[2] P. J. Davis and P. Rabinowitz, Methods of Numerical Integration, 2nd ed., Academic Press, Orlando, 1984. MR 760629 (86d:65004)

[3] K. C. Chung, G. A. Evans and J. R. Webster, A method to generate generalized quadrature rules for oscillatory integrals, Appl. Numer. Math. 34 (2000), 85-93. MR.1755695 (2002a:65048)

[4] G. A. Evans and K. C. Chung, Some theoretical aspects of generalised quadrature methods, J. Complexity 19 (2003), 272-285. MR.1984114 (2004c:41064)

[5] L. N. G. Filon, On a quadrature formula for trigonometric integrals, Proc. Royal Soc. Edinburgh 49 (1928), 38-47.

[6] E. A. Flinn, A modification of Filon's method of numerical integration, J. Assoc. Comput. Mach. 7 (1960), 181-184. MR0114298 (22:5122)

[7] I. S. Gradshteyn and I. M. Ryzhik, Table of Integrals, Series, and Products, 5th ed., Academic Press, Boston, MA, 1994. MR1243179 (94g:00008)

[8] D. Huybrechs and S. Vandewalle, On the evaluation of highly oscillatory integrals by analytic continuation, SIAM J. Numer. Anal. 44 (2006), 1026-1048. MR2231854 (2007d:41033) 
[9] D. Huybrechs and S. Vandewalle, A sparse discretization for integral equation formulations of high frequency scattering problems, SIAM J. Sci. Comput., 29 (2007), 2305-2328. MR.2357616 (2008g:65178)

[10] A. Iserles and S. P. Nørsett, On quadrature methods for highly oscillatory integrals and their implementation, BIT 44 (2004), 755-772. MR2211043 (2006k:65060)

[11] A. Iserles and S. P. Nørsett, Efficient quadrature of highly oscillatory integrals using derivatives, Proc. Royal Soc. A 461 (2005), 1383-1399. MR2147752 (2006b:65030)

[12] A. Iserles, S. P. Nørsett and S. Olver, Highly oscillatory quadrature: The story so far, Proceedings of ENUMATH, Santiago de Compostela (2005) (A. Bermudez de Castro et al., eds.), Springer-Verlag, Berlin, 2006, 97-118. MR 2303638 (2008j:65029)

[13] A. Iserles and S. P. Nørsett, Highly oscillatory quadrature and its applications, http://handle. dtic.mil/100. 2/ADA433730, Defense Technical Information Center, 2005.

[14] D. Levin, Procedures for computing one- and two-dimensional integrals of functions with rapid irregular oscillations, Math. Comp. 38 (1982), 531-538. MR645668 (83a:65023)

[15] D. Levin, Fast integration of rapidly oscillatory functions, J. Comput. Appl. Math. 67 (1996), 95-101. MR.1388139 (97a:65029)

[16] Y. K. Luke, On the computation of oscillatory integrals, Proc. Cambridge Philos. Soc. 50 (1954), 269-277. MR0062518 (15:992b)

[17] S. Olver, Moment-free numerical integration of highly oscillatory functions, IMA J. Numer. Anal. 26 (2006), 213-227. MR2218631(2006k:65064)

[18] S. Olver, Numerical approximation of vector-valued highly oscillatory integrals, BIT 47 (2007), 637-655. MR2338536(2008i:65043)

[19] S. Olver, Moment-free numerical approximation of highly oscillatory functions with stationary points, Euro. J. Appl. Math. 18 (2007), 435-447. MR2344314 (2008g:65045)

[20] R. Piessens, Automatic computation of Bessel function integrals, Comput. Phys. Commun. 25 (1982), 289-295.

[21] R. Piessens and M. Branders, Modified Clenshaw-Curtis method for the computation of Bessel function integrals, BIT 23 (1983), 370-381. MR705003 (85b:65019)

[22] E. Stein, Harmonic Analysis: Real-variable methods, orthogonality, and oscillatory integrals, Princeton University Press, Princeton, 1993. MR,1232192(95c:42002)

[23] L. N. Trefethen, Is Gauss quadrature better than Clenshaw-Curtis, SIAM Review 50 (2008), 67-87. MR2403058 (2009c:65061)

[24] G. N. Watson, A Treatise on the Theory of Bessel Functions, Cambridge University Press, Cambridge, 1952. MR1349110 (96i:33010)

[25] S. Xiang, Efficient Filon-type methods for $\int_{a}^{b} f(x) e^{i \omega g(x)} d x$, Numer. Math. 105 (2007), 633658. MR.2276763 (2008k:65051)

[26] S. Xiang, Numerical analysis of a fast integration method for highly oscillatory functions, BIT 47 (2007), 469-482. MR2334051 (2008e:65096)

[27] S. Xiang, W. Gui and P. Mo, Numerical quadrature for Bessel transformations, Appl. Numer. Math. 58 (2008), 1247-1261. MR2444255 (2009e:65047)

[28] S. Xiang and W. Gui, On generalized quadrature rules for fast oscillatory integrals, Appl. Math. Comp. 197 (2008), 60-75. MR 2396291

Department of Applied Mathematics and Software, Central South University, Changsha, Hunan 410083, People's Republic of China

Current address: Department of Applied Mathematics, The Hong Kong Polytechnic University, Hung Hom, Kowloon, Hong Kong

Department of Applied Mathematics and Software, Central South University, Changsha, Hunan 410083, People's Republic of China 